\title{
Does the Different Branching Direction Affect Memory Accuracy?
}

\author{
Qijun Feng ${ }^{1, *, \dagger}$, Yunzi Gai ${ }^{2,{ }^{*}, \dagger}$, Jiacheng $\mathrm{Hao}^{3, *}{ }^{*}$, \\ ${ }^{1}$ Shanghai Theatre Academy, Shanghai, China \\ ${ }^{2}$ University of Vermont, Burlington, U.S.A. \\ ${ }^{3}$ University of Toronto, Toronto, Canada \\ ${ }^{*}$ Corresponding author.Email: guanghua.ren@gecacdemy.cn
}

These authors contributed equally.

\begin{abstract}
The relationship between language and memory has always been a subject of numerous researches. One hypothesis is that language affects how we perceive the world. Recent studies have found that the branching direction of a person's native language affects his/her working memory and the way that he/she process information. Based on previous research, this research aimed to study the effect of the branching direction of non-native languages on working memory. Forty-six adult native speakers of different linguistic groups (LB \& RB) were assigned to participate in two different working memory tasks. Compared to LB speakers, RB speakers are better at recalling final stimuli but worse at recalling initial stimuli in both tasks of object recalling and number recalling $(\mathrm{P}<0.05)$. This result is consistent with the pattern of the previous study. It suggests that the use of non-native languages with different branching directions will not significantly change the impact of the native language on our working memory.
\end{abstract}

Keywords: Memory, Language, Whorf hypothesis

\section{INTRODUCTION}

Language and memory are two cognitive functions that are undoubtedly extremely important for us to function normally. However, the relationship between the two has always been complicated and controversial throughout the history of psychology. In the early ages, they have been studied as two cognitive functions that have little influence on each other [1]. More research in related fields has been conducted, the Whorf hypothesis (linguistic relativity) appeared [2]. It states that the structure of a person's spoken language will affect his cognition and perceptions of the real world. Those who speak different languages would have different ways of thinking [3]. However, there are not only scholars who challenge this hypothesis, but there are also disagreements among those who support this theory, mainly about the degree of language's influence on advanced cognitive functions. Some people think that language determines people's thoughts and cognition. But this kind of idea is usually considered incorrect is no longer accepted by modern psychologists [4]. The other group believes that language only influences our way of thinking [5].

To gain a better understanding of the relationship between memory and language. In the past decade, many psychologists have designed different experiments to study the impact of many aspects of language on human memory and cognition. These include pronunciation, intonation, sentence structure, and syntax. One of the most popular ideas in related literature is that the structural features of one's native language would significantly influence his working memory. In 2019, Federica Amici and her colleagues at the University of Leipzig had conducted an experiment to test this hypothesis. They have specifically focused on the impact of the word order or the branching direction of one's native language [6]. In linguistics, word order, or syntax, is called branching direction. The left-branching language LB stands for Chinese and Japanese. In these languages, modification usually precedes the heads. In right-branching languages, such as Italian and Spanish, the heads are at the beginning of the sentence. Amici and his colleagues believe that such differences will 
have an essential impact on our working memory and thus on higher-order cognitive functions.

Their reason is that we use language every day, getting used to such cognitive processing patterns. For LB people, often need to wait until the end of the sentence to know what is being said, so they need to temporarily store information in working memory. So, for a series of information, their ability to remember the initial stimuli may be better than the RB people, and the $\mathrm{RB}$ people will perform better in recalling the stimuli at the end.

One common strategy used to study working memory is to use memory recognition tests. This applies to the experiment of Amici and her colleagues. Their setting contained short and working memory tasks. The participants were asked to remember the several stimuli presented in each trial and then recall them in the same order. The results were promising and have confirmed their hypothesis. The experiment has significant meanings in the field because these huge influences on the sequential processing of information caused by the language's syntactic structures have revealed the influence of the language of memory. Also, cognition is not limited to conceptual or semantic biases but rather to a larger, non-linguistic part [2]. It could affect how we process information and therefore possess tremendous influences on our higher cognitive functions such as problem-solving and decision-making [6]. It's also further proof of the Whorf hypothesis [2].

However, there is a problem with their experiment that the encoding and retrieval parts were all performed in the native language of the participants. What if they use a non-native language? The result is still unknown. With more and more studies on bilingualism or trilingualism, recent theoretical developments have shown that the use of non-native languages can have a huge and multifaceted impact on cognitive abilities in a person's life [7]. Regarding its relationship with working memory, many studies have examined whether the second or third language will have a profound effect on working memory, e.g. [8-10]. A meta-analysis conducted by Linck and his colleagues at the University of Maryland showed that working memory has a robust relationship with second languages indeed [11].

As far as we know, no previous research has investigated the effect of the non-native languages' branching directions on the working memory. Going back to the branching direction, if participants use a non-native language, will they be affected by the new language, or will they maintain the patterns of their native languages. To address this question, we have conducted an experiment containing working memory tasks that were performed completely in different languages different from the participant's native language. We, therefore, analysed the effect of nonnative languages on working memory and whether it can change the cognitive pattern set by our mother languages. It could provide a more sophisticated study of the Whorf hypothesis in the field of non-native languages. Our hypothesis is those non-native languages will not have a significant impact on participants' performance. Because the native language is learned from birth, it can be said that its influence is deep-rooted. And for many participants, even if they use a non-native language to test, they still use their native language in their hearts.

\section{METHODS}

\subsection{Participants}

Forty-six participants voluntarily took part in the experiment. They participated in the experiment through the online platform Pavlovia of PsychoJS/Psychopy. They speak different native languages and can fluently use English, which is their second or third language. Most of the participants, ranging in age from 18 to 42 years old of both genders, are students or researchers in higher education. According to the branching direction of their native languages, 24 participants (age: $\mathrm{M}=$ 25.54; $\mathrm{SD}=5.37$ ) were divided into the Left-Branching group, which included users of four different languages (12 Chinese, 6 Japanese, 5 Korean, and 1 Basque). The remaining 22 participants (age: $\mathrm{M}=26.73$; $\mathrm{SD}=6.40$ ) were divided into the Right -Branching group, which contains users of five different languages (7 Italian, 5 Spanish, 4 French, 4 Indonesian, and 2 Thai).

All participants have normal language skills, normal or correct-to-normal vision. All participants reported no history of mental illness or disorders. All participants are aware of this experiment and agree to volunteer. The local ethics committee approved this study.

\subsection{Materials}

In each task, 50 pictures $(600 \mathrm{px} \times 800 \mathrm{px})$ were used. In task 1,50 pictures of objects were displayed. They are selected from a set of free materials on the internet. They were composed of pictures of different objects, including food, electronic products, vehicles, etc. They were universal and easily recognizable through different cultures. Task 2 contained 50 pictures of English letters that we made. In each picture, the number, position, and colour of different English letters were different. All stimuli were presented in the middle of the computer screen, and the background colour was light grey.

\subsection{Procedure}

All our experiments were conducted online, so the experiments took place in environments familiar to the participants. We required the experimental environment to be quiet and free of other interference. The computers 
should be normal functioning, and the experiment should be running on full screen.

In task 1 , the participants were presented with 12 trials, each with 10 stimuli $(600 \mathrm{px} \times 800 \mathrm{px}$ images of objects). They were instructed before the task to remember those objects in the same order as they were presented. Fig 1 showed the main procedures in this experiment. The trials began with a fixation in the middle of the computer screen for $2000 \mathrm{~ms}$, and then 10 stimuli were displayed in the middle of the screen, each being visible for $2000 \mathrm{~ms}$ and had a $500 \mathrm{~ms}$ break in between. After the presentation of stimuli, the participants were asked to type down the objects they have seen in English and in the same order.
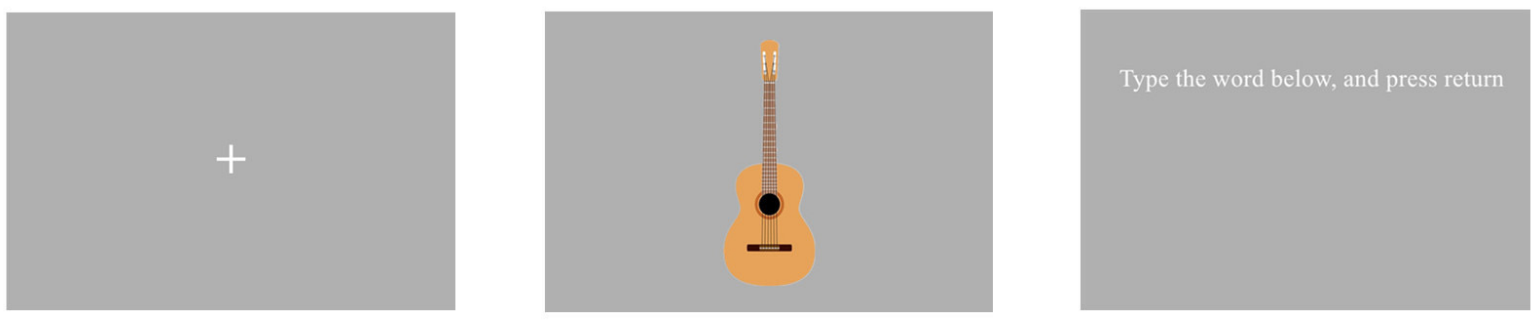

$2000 \mathrm{~ms}$

10 stimuli in total $2000 \mathrm{~ms}$ for each $500 \mathrm{~ms}$ break in between

$2000 \mathrm{~ms}$

$500 \mathrm{~ms}$ break

$500 \mathrm{~ms}$ break

Figure 1. Task 1

In task 2, the participants were presented with 12 trials, each with 10 stimuli $(600 \mathrm{px} \times 800 \mathrm{px}$ images of English letters). They were instructed before the task to count the number of a specific letter and remember those numbers in the same order as they were presented. Fig 2 showed the main procedures in this experiment. The trials began with a fixation in the middle of the computer screen for $2000 \mathrm{~ms}$, and then 10 stimuli were displayed in the middle of the screen, each being visible for $2000 \mathrm{~ms}$ and had a $500 \mathrm{~ms}$ break in between. After the presentation of stimuli, the participants were asked to type down the numbers they remembered in English and in the same order.

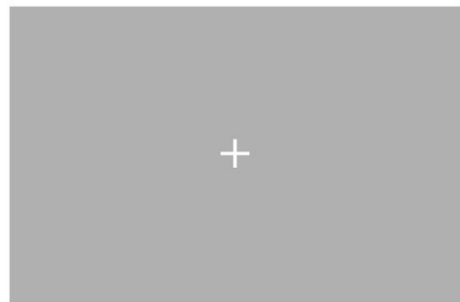

$2000 \mathrm{~ms}$

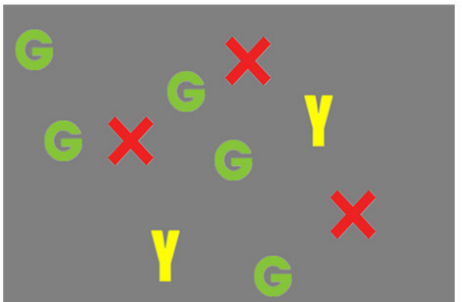

10 stimuli in total $2000 \mathrm{~ms}$ for each $500 \mathrm{~ms}$ break in between

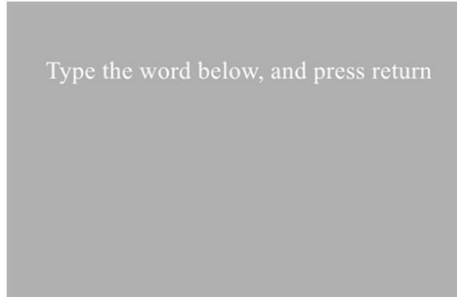

$2000 \mathrm{~ms}$

Figure 2. Task 2

\section{RESULTS}

\subsection{The effects of branching on WM Task 1.}

The comparison of the performances between leftbranching and right-branching showed significant differences in task $1(\mathrm{p}<0.001, \mathrm{~N}=46)$. Independent samples t-test was used in this experiment to study the differences of different branching types for the scores collected for initial and final performances. Different language branching types were significantly affected by performances on recalling initial stimuli and final stimuli $(p<0.05)$. LB participants were better at recalling initial stimuli than RB participants, and RB participants were better at recalling final stimuli. Table 1 shows that different branching types indicated a significant level of 0.01 for Initial Score $(t=9.339, p=0.000)$, and the specific comparison showed that the mean value of $L B$ $(M=3.50)$ was significantly higher than the $R B$ average $(\mathrm{M}=2.60)$. Different branching types indicated a significant level of 0.01 for Final Score $(t=-7.777$, $\mathrm{p}=0.000$ ), and the specific comparison showed that the mean value of $L B(M=2.69)$ was significantly below $R B$ $(\mathrm{M}=3.55)$.

Table 1. The Effects of Branching in WM Task 1

\begin{tabular}{llllll}
\hline & Branching & Mean & SD & $\boldsymbol{t}$ & $\boldsymbol{p}$ \\
\hline Initial & Left $(\mathrm{n}=24)$ & 3.51 & 0.42 & 8.255 & $0.000 * *$ \\
Score & Right $(\mathrm{n}=22)$ & 2.59 & 0.33 & & \\
\hline Final & Left $(\mathrm{n}=24)$ & 2.55 & 0.39 & - & $0.000 *$ \\
Score & & & & 8.524 & \\
& Right $(\mathrm{n}=22)$ & 3.42 & 0.30 & & \\
\hline$* \mathrm{p}<0.05 * * \mathrm{p}<0.01$ & & & &
\end{tabular}




\subsection{The Effects of Branching on WM Task 2}

The comparison of the performances between leftbranching and right-branching showed significant differences in task $2(\mathrm{p}<0.001, \mathrm{~N}=46)$. Independent samples t-test was used in this experiment to study the differences of different branching types for the scores collected for initial and final performances. Different language branching types were significantly affected by performances on recalling initial stimuli and final stimuli $(p<0.05)$. LB participants were better at recalling initial stimuli than RB participants, and RB participants were better at recalling final stimuli. Table 2 shows that different branching types indicated a significant level of 0.01 for Initial Score $(\mathrm{t}=8.255, \mathrm{p}=0.000)$, and the specific comparison showed that the mean value of LB $(M=3.51)$ was significantly higher than the $R B$ average $(\mathrm{M}=2.59)$. Different branching types indicated a significant level of 0.01 for Final Score $(t=-8.524$, $\mathrm{p}=0.000$ ), and the specific comparison showed that the mean value of $\mathrm{LB}(\mathrm{M}=2.55)$ was significantly below $\mathrm{RB}$ $(\mathrm{M}=3.42)$.

Table 2. The Effects of Branching in WM Task $2 * \mathrm{p}<0.05 * * \mathrm{p}<0.01$

\begin{tabular}{llllll}
\hline & Branching & Mean & SD & $\boldsymbol{t}$ & $\boldsymbol{p}$ \\
\hline Initial Score & Left $(\mathrm{n}=24)$ & 3.50 & 0.30 & 9.339 & $0.000 * *$ \\
& Right $(\mathrm{n}=22)$ & 2.60 & 0.35 & & \\
\hline Final Score & Left $(\mathrm{n}=24)$ & 2.69 & 0.41 & -7.777 & $0.000 * *$ \\
& Right $(\mathrm{n}=22)$ & 3.55 & 0.32 & & \\
\hline
\end{tabular}

\section{DISCUSSION}

As predicted, the current study explored that LB speakers were better at recalling initial stimuli while RB speakers were better at recalling final stimuli. We found that the results produced by the two working memory tasks corresponded to our hypothesis, which is that nonnative languages will not significantly impact people's memory and cognitive functions. In other words, the branching direction of their native language played a decisive role in memory accuracy. In this research, the non-native language we chose is English. The branching direction of English is right; however, RB participants did not perform better than LB participants, and there was no significant difference in scores between RB and LB participants. Compared with the native language, no matter what their second language is, the branching direction of the native language determined people's memory accuracy, in other words, working memory and cognitive functions [3]. Therefore, this aspect of the research suggested that non-native languages with different branching directions will not substantially change working memory. The result was similar to those of previous experiments [6], in line with their branching directions. It further proves that the relationship between language and cognitive ability is about conceptual representations and semantic biases, and the sequential processing of information and sentence structures. The position of different subjects in a sentence affects how we acquire, process, and recall information. And the ability to maintain that information in working memory is even more important because it is the basis of many higher cognitive functions, such as problem-solving and decision-making. Native language is the language that people learn from a young age, and it has a very deep and long-lasting effect on people's memory. Although people can choose to learn a second, third, or even fourth language later in life, these later languages require longer and more immersive use if they are to have a larger impact on memory than their native language.

However, like any other study, this study is not free from limitations. First, the small sample size- limits the generalization. Because of the specificity of our participants, native speakers of different languages who are fluent in English, it makes the process of finding participants even more difficult. More data and more different languages are needed to verify the results in the current study. Another important question is what language do the participants use in their minds during the encoding and retrieval process [10]. In future experiments, it is necessary to ask these questions through questionnaires at the end of the experiment. If they still use their native languages, then it can further support our conclusion. Finally, everyone's mastery of the second or third language is different. Therefore, what also needs to consider the length of time they live in an English-speaking country and the length of time they study English [7]. There are a number of factors that we need to consider when we're looking at participants' English proficiency so that we can get a clearer picture of how their English affects their memory [9]. Because this experiment did not include the part about these issues, in the subsequent experimental design, consideration of these elements can be added to study the correlation between the impact of non-native language on working memory and the degree of mastery of this language.

The second is the task design. Future experiments should be designed to measure the working memory of participants by designing tasks with different stimuli. In subsequent experiments, include more different tasks, short-term test memory, and working memory separately and add more trails to each task. This gives a more accurate view of the working memory capacity of speakers speaking different languages. 
Taken together, this study provides some important practical implications for the relationship between language and thought. It is a good confirmation that language has a fundamental impact on human cognitive abilities and that biologically relevant behaviours engaging higher cognitive functions can drastically affect our memory and even cause long-term structural changes to our brain [6]. Also, we are clearly aware of the importance of quantifying the participants' language level, which is important to influence the results of the experiment. So, it still needs to discuss and research more for the relationship between language and memory.

\section{CONCLUSION}

To sum up, the relationship between language and memory is still controversial and complex nowadays. Our study aimed to find the mechanism between language and memory because the language we used every day had a great impact and changed our working memory and cognitive functions. To our knowledge, this is the first study that explores the effect of a second or third language on working memory. It is an attempt in this direction and can provide support for research in the field of language and memory. In addition, with the development of technology and education levels, the number of bilinguals and even trilinguals is increasing year by year. People no longer speak only their native language in daily life. This raised many unknown questions, such as whether learning and using multiple languages changes the structure of the human brain? Are bilinguals or trilinguals better at remembering than monolinguals? Although our experiment is small, linguists and medics may draw inspiration from our studies and help them learn more about the relationship between language and memory and solve more possible problems related in the future. The contribution made here has wide applicability and should be of wide interest in further research in this field.

\section{REFERENCES}

[1] H. Ebbinghaus, Memory: A contribution to experimental psychology, 1964.

[2] E. Hunt, F. Agnoli, The Whorfian hypothesis: A cognitive psychology perspective. Psychological Review, 1991, 98(3), 377-389.

[3] J. Leavitt, Linguistic Relativities: Language Diversity and Modern Thought. (n.p.): Cambridge University Press, 2010.

[4] Ahearn, M. Laura, Living language : an introduction to linguistic anthropology. Chichester, West Sussex, U.K, 2012, p. 69. ISBN 978-1-44434056-3. OCLC 729731177.

[5] H. J. Ottenheimer, J. M. Pine, The Anthropology of Language: An Introduction to Linguistic
Anthropology. United States: Cengage Learning, 2018.

[6] F. Amici, A. Sánchez-Amaro, C. Sebastián-Enesco, T. Cacchione, M. Allritz, J. Salazar-Bonet, F. Rossano, The word order of languages predicts native speakers' working memory. Scientific reports, 2019, 9(1), 1-12.

[7] E. Bialystok, G. Poarch, Language Experience Changes Language and Cognitive Ability, Zeitschrift fur Erziehungswissenschaft : ZfE. 2014, https://www.ncbi.nlm.nih.gov/pmc/articles/PMC42 $43303 /$.

[8] A. Juffs, M. Harrington, Aspects of working memory in L2 learning: Language Teaching. Cambridge Core, 2011, https://www.cambridge.org/core/journals/language -teaching/article/abs/aspects-of-working-memoryin-12-

learning/F7FDA033F0E3931732B8AE03D3E2C3 36.

[9] A.-M. Adams, K. Guillot, Working Memory and Writing in Bilingual Students. ITL - International Journal of Applied Linguistics, 2008, https://www.jbeplatform.com/content/journals/10.2143/ITL.156.0. 2034417.

[10] C. A. Sanchez, J. Wiley, T. K. Miura, G. J. H. Colflesh, T. R. Ricks, M. S. Jensen, A. R. A. Conway, Assessing working memory capacity in a non-native language. Learning and Individual Differences, 2010 , https://www.sciencedirect.com/science/article/abs/ pii/S1041608010000361.

[11] J. A. Linck, P. Osthus, J. T. Koeth, M. F. Bunting, Working memory and second language comprehension and production: A meta-analysis. Psychonomic Bulletin \& Review, 2013, https://link.springer.com/article/10.3758/s13423013-0565-2. 